\title{
Gastric metaplasia of the duodenal mucosa in Crohn's disease: novel histological and endoscopic findings
}

\section{다)(웅}

\author{
Authors

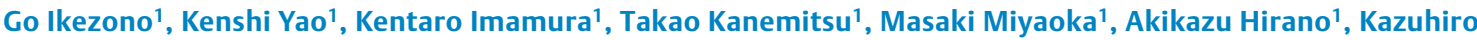 \\ Takeda', Takashi Hisabe ${ }^{1}$, Toshiharu Ueki ${ }^{1}$, Hiroshi Tanabe², Atsuko Ota², Seiji Haraoka², Akinori Iwashita²
}

Institutions

1 Department of Endoscopy, Fukuoka University Chikushi Hospital

2 Department of Pathology, Fukuoka, University Chikushi Hospital

submitted 1.7.2020

accepted after revision 8.10 .2020

Bibliography

Endoscopy International Open 2021; 09: E181-E189

DOI 10.1055/a-1313-7239

ISSN 2364-3722

(C) 2021. The Author(s).

This is an open access article published by Thieme under the terms of the Creative Commons Attribution-NonDerivative-NonCommercial License, permitting copying and reproduction so long as the original work is given appropriate credit. Contents may not be used for commecial purposes, or adapted, remixed, transformed or built upon. (https://creativecommons.org/licenses/by-nc-nd/4.0/)

Georg Thieme Verlag KG, Rüdigerstraße 14,

70469 Stuttgart, Germany

Corresponding author

Kenshi Yao, MD, PhD, Department of Endoscopy, Fukuoka University Chikushi Hospital, Zokumyoin, Chikushino City,

Fukuoka 818-8502, Japan

Fax: +81-(0)92-929-2630

yao@fukuoka-u.ac.jp

\section{ABSTRACT}

Background and study aims Upper gastrointestinal endoscopy and biopsy are useful for differential diagnosis of Crohn's disease (CD) of the large intestine and ulcerative colitis (UC). We aimed to identify novel histopathological and endoscopic findings in the upper gastrointestinal tract in patients with CD who did not have Helicobacter pylori infection.

Patients and methods Upper gastrointestinal endoscopy was performed on patients with CD and UC. Mucosal lesions detected were subsequently observed using magnifying endoscopy with narrow-band imaging (M-NBI), following which biopsy was performed. When no mucosal lesion was detected on conventional endoscopy, M-NBI and biopsy were performed on four sites: the gastric body, gastric antrum, duodenal bulb, and second portion of the duodenum. Results The prevalences of gastric metaplasia (GM) were $48 \%(24 / 50)$ and $16 \%(8 / 50)$ in the CD and UC groups, showing a significant difference $(P=0.001)$. In 23 of 24 patients with histologically proven GM in the CD group, mucosal lesions were detected using conventional white-light imaging (C-WLI). In 22 of 24 patients with histologically proven GM in the CD group, disappearance of normal villous structure and the presence of curved marginal crypt epithelium were noted using magnifying endoscopic findings characteristic of GM (M-GM). A combination of C-WLI and $\mathrm{M}-\mathrm{NBI}$ yielded a significantly increased specificity $(P=$ $0.004)$ and accuracy $(P=0.039)$.

Conclusions The prevalence of GM in the duodenal mucosa was significantly higher in patients with $C D$ than in controls. The identified endoscopic findings may be useful as novel indicators for the histological diagnosis of GM in the duodenum.

\section{Introduction}

Chronic inflammatory bowel diseases are broadly classified into Crohn's disease (CD) and ulcerative colitis (UC). CD is characterized by upper gastrointestinal lesions [1-4]. In addition, the characteristic histological findings of $C D$ have been reported to include noncaseating epithelioid granulomas [5] and the pres- ence of focally enhanced gastritis in the gastric mucosa without Helicobacter pylori (HP) infection [6]. These characteristic endoscopic and histological findings of the upper gastrointestinal mucosa in $C D$ are useful in the differential diagnosis of $C D$ of the large intestine and UC [1-4]. However, the detection rate for noncaseating epithelioid granuloma based on histological examination of biopsy specimens collected during upper gas- 
trointestinal endoscopy has been decreasing in recent years according to our recent experience. In our experience of evaluating the histopathological findings of endoscopically biopsied specimens from the gastroduodenal mucosa of patients with $C D$, we have frequently detected gastric metaplasia $(G M)$ in the duodenum and intestinal metaplasia in the stomach. Further, a previous study has reported that GM in the duodenum is one of the characteristic histological findings of CD, distinguishing it from Behçet's disease [7]. However, whether these histologic findings can serve as markers for differentiating $C D$ from UC has not yet been investigated. Thus, it may be meaningful to identify novel histological and endoscopic indicators that could serve as useful novel markers for the differential diagnosis of CD and UC. We designed and conducted a study to identify the characteristic histopathological findings in the upper gastrointestinal tract and the characteristic endoscopic findings in the upper gastrointestinal mucosa in patients with CD who do not have HP infection.

\section{Patients and methods}

In this retrospective study, we enrolled 50 consecutive patients with CD who underwent upper gastrointestinal endoscopy with a magnifying endoscope in the Department of Gastroenterology, Fukuoka University Chikushi Hospital, between April 2018 and March 2020. Consecutive 50 Patients with UC during the same period were included as controls.

\section{Inclusion criteria}

Inclusion criteria were as follows: 1 . Use of upper gastrointestinal endoscopy with a magnifying endoscope for diagnosing $C D$ or UC; 2 . Absence of HP infection, defined as presence of endoscopic findings characteristic of an HP-uninfected stomach [8, 9], absence of a history of HP eradication, and negative results on at least two diagnostic tests for HP infection (HP immunoglobulin $G$ antibody test, urea breath test, stool antigen test, rapid urease test, culture of biopsy specimens, and microscopy of biopsy specimens); 3. Eastern Cooperative Oncology Group performance status score of 0 or 1 [10].

\section{Exclusion criteria}

Exclusion criteria were as follows: An indication of endoscopy for diagnosing an upper gastrointestinal hemorrhage, postoperative stomach or reconstruction of a gastric tube, presence of a serious underlying disease, presence of concurrent psychiatric diseases or symptoms that would make participation in the study difficult, participation deemed inappropriate by the principal investigator or sub-investigators.

\section{Endoscopic procedures and biopsy}

A specialist in Gastrointestinal Endoscopy certified by the Japanese Gastroenterological Endoscopy Society or an equally qualified endoscopist with more than 10 years of experience in endoscopy performed the procedures.

In this study, endoscopy was performed as follows. First, conventional white-light imaging (C-WLI) was performed to determine the presence or absence of mucosal lesions. When
C-WLI revealed mucosal lesions, M-NBI was performed, and target biopsies at the observation sites were performed. When no mucosal lesion was detected, magnifying endoscopy with narrow-band imaging (M-NBI) and biopsy of the gastric body, gastric antrum, duodenal bulb, and second portion of the duodenum were randomly performed.

\section{Histopathological assessment}

Biopsy specimens were fixed in $20 \%$ formalin and embedded in paraffin. Sections of the specimens were stained with hematoxylin and eosin (HE) and Alcian blue-periodic acid Schiff (AB-PAS) stain. We examined histopathological characteristics in these stained specimens.

\section{Histopathological definition of gastric metaplasia in the duodenum}

Replacement of the absorptive epithelium of the small intestine with the gastric crypt epithelium on HE staining was used to define GM. In addition, intense staining of the entire cells and reddish-purple staining of the mucosal epithelium replaced with the gastric crypt epithelium on AB-PAS staining were performed to confirm GM $[11,12]$. Ectopic gastric mucosa associated with proper gastric glands were not used to define GM in this study.

\section{Histopathological definition of intestinal metaplasia in the stomach}

Intestinal metaplasia was defined as the presence of goblet cells in the gastric epithelium on HE staining, which were stained blue with the AB-PAS stain.

\section{Review of endoscopic findings}

An independent endoscopist who was unaware of the patients' histopathological findings reviewed the endoscopic findings (CWLI and $\mathrm{M}-\mathrm{NBI}$ ). In this study, endoscopic findings of C-WLI were classified by color (red, normal, and pale) and morphology (protruded, flat, depressed). As for the endoscopic M-NBI findings, the anatomical findings visualized by $\mathrm{M}-\mathrm{NBI}$ were analyzed.

\section{Endpoints}

The primary endpoint involved the characteristic histopathological findings of the upper gastrointestinal tract in patients with CD who did not have HP infection. The secondary endpoints included the following: Prevalence of mucosal lesions and characteristic endoscopic findings; prevalence of mucosal lesions exhibiting GM; magnifying endoscopic findings characteristic of gastric metaplasia (M-GM); diagnostic performances (sensitivity, specificity, and accuracy) of C-WLI and C-WLI+M-NBI in the detection of GM; prevalence of GM in the duodenum according to the presence or absence of a bamboo joint-like appearance and duodenal notch; and prevalence of histologically proven $\mathrm{GM}$ and M-GM by the type of CD. 
- Table 1 Patient characteristics.

\begin{tabular}{|c|c|c|c|}
\hline & Crohn's disease $(n=50)$ & Ulcerative colitis $(n=50)$ & $P$ value \\
\hline Age (years) (mean $\pm S D$ ) & $37.8 \pm 15.0$ & $41.9 \pm 16.1$ & $0.189^{1}$ \\
\hline Sex (male/female) & $33 / 17$ & $28 / 22$ & $0.412^{2}$ \\
\hline Disease duration (month) (mean \pm SD) & $144.3 \pm 135.4$ & $98.9 \pm 112.2$ & $0.071^{1}$ \\
\hline $\begin{array}{l}\text { SD, standard deviation. } \\
1 \text { Student's t-test } \\
{ }^{2} \text { Fisher's exact test }\end{array}$ & & & \\
\hline
\end{tabular}

- Table 2 Histological findings in the duodenal mucosa.

\begin{tabular}{|l|l|l|l|}
\hline & Crohn's disease $\mathbf{( n = 5 0 )}$ & Ulcerative colitis (n= 50) & P value \\
\hline Gastric metaplasia & $24(48 \%)$ & $8(16 \%)$ & 0.001 \\
\hline Noncaseating granuloma & $1(2 \%)$ & $0(0 \%)$ & n.s. \\
\hline No specific findings & $25(50 \%)$ & $42(84 \%)$ & n.s. \\
\hline n.s., not significant; Fisher's exact test. & & & \\
\hline
\end{tabular}

\section{Interobserver and intraobserver agreement}

Endoscopic images taken by C-WLI and $\mathrm{M}-\mathrm{NBI}$ of 50 patients with CD and 50 patients with UC were obtained to test interobserver agreement. The images were arranged in random order. First, to determine interobserver agreement, two independent endoscopists with 14 (K. I.) and 10 years (G. I.) of experience in gastric endoscopy were asked to review all images and determine whether each image showed the presence or absence of mucosal lesions (C-WLI) and M-GM (M-NBI) [13]. To determine intraobserver agreement, the first endoscopist was asked to review all the images 2 weeks later and to determine whether each image showed the presence or absence of mucosal lesions and M-GM [13].

\section{Statistical analysis}

Comparisons between the two groups were performed using Fisher's exact test, McNemar's test, and Bonferroni correction, while a comparison of mean values between the groups was performed using an unpaired Student's $t$-test. $P<0.05$ was considered to indicate statistical significance in all tests. Kappa values were calculated for interobserver and intraobserver agreement. Agreement was classified as excellent for $\geq 0.8$; good for $<0.8$ to $\geq 0.6$; moderate for $<0.6$ to $\geq 0.4$; and fair for $<0.4$. SPSS version $21 \mathrm{~J}$ for Windows (SPSS, Chicago, Illinois, United States).

\section{Ethical considerations}

This study was conducted in accordance with the 1964 Declaration of Helsinki and its subsequent amendments. Written informed consent was obtained from each patient. Personal information listed in the medical records or obtained through questionnaire surveys, including data on informed consent, was managed in a database such that individual data were anonymized. This study was approved by the institutional review board of Fukuoka University Chikushi Hospital (C2001-006).

\section{Results}

There were no significant differences in age, sex, or medical history between the CD and UC groups ( $\downarrow$ Table 1 ). In the CD group, based on the Crohn's Disease Activity Index, the disease activity level was assessed as inactive in 34 patients, active in 15 , and severe in one; the distribution of the disease type was as follows: the small and large intestines in 33 patients, small intestine in eight, and large intestine in nine. In the UC group, based on the Sutherland Index, the disease activity was assessed as mild in 28 patients, moderate in 20, and severe in two; the distribution of the disease type was as follows: the pancolitis type in 37 patients, left colitis type in 10, and proctitis type in three.

\section{Primary endpoint}

On histological examination of the biopsy specimens collected from duodenal mucosae, GM was detected in $48 \%$ of patients $(24 / 50)$ in the CD group and $16 \%$ of patients $(8 / 50)$ in the UC group; a significant difference was observed $(P=0.001)$ ( $>$ Table 2 ). In addition, noncaseating granulomas in the duodenum were detected only in $2 \%$ of patients $(1 / 50)$ in the CD group.

On histological examination of the biopsy specimens collected from HP-negative gastric mucosae, intestinal metaplasia was detected in $8 \%$ of patients $(4 / 50)$ in the CD group and $6 \%$ of those $(3 / 50)$ in the UC group; the difference was not significant $(P=1.000)$ ( $\triangleright$ Table 3$)$. Focally enhanced gastritis was detected in $18 \%$ of patients $(9 / 50)$ in the CD group but was not detected in the UC group ( $>$ Table 3 ). In addition, noncaseating granulomas in the stomach were detected only in one $(2 \%)$ of 50 patients in the CD group ( $\triangleright$ Table 3 ). 
$>$ Table 3 Histological findings in gastric mucosa.

\begin{tabular}{|l|c|c|c|}
\hline & Crohn's disease $(\mathbf{n = 5 0 )}$ & Ulcerative colitis (n=50) & P value \\
\hline Intestinal metaplasia & $4(8 \%)$ & $3(6 \%)$ & n.s. \\
\hline Focally enhanced gastritis & $9(18 \%)$ & $0(0 \%)$ & $<0.001$ \\
\hline Noncaseating granuloma & $1(2 \%)$ & $0(0 \%)$ & n.s. \\
\hline No specific findings & $36(72 \%)$ & $47(94 \%)$ & n.s. \\
\hline n.s., not significant; Fisher's exact test. & & & \\
\hline
\end{tabular}
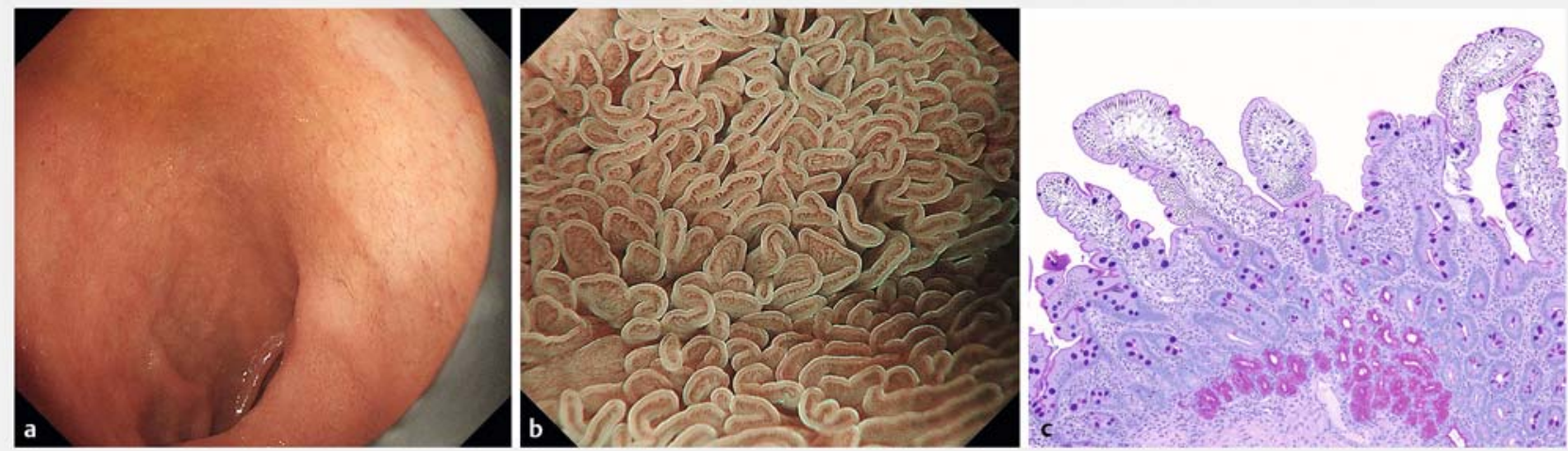

- Fig. 1 Endoscopic and histopathological findings of a normal duodenum. a Duodenal bulb: normal mucosa. b Magnifying endoscopy with narrow-band imaging at the same site. The normal villous structure is preserved. $\mathbf{c}$ Histopathological findings of the same site. Alcian blueperiodic acid Schiff staining showing normal duodenal mucosa.
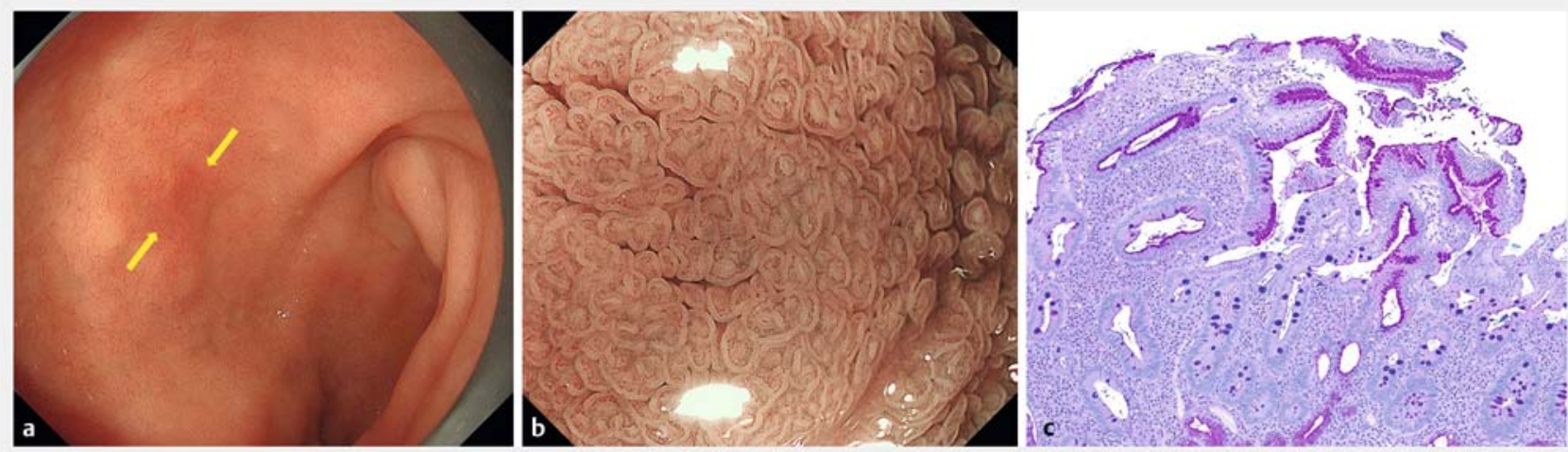

- Fig. 2 Endoscopic and histopathological findings of the duodenum with mucosal lesions. a Conventional white-light imaging. Red flat lesions are observed in the duodenal bulb (arrow). b Magnifying endoscopy with narrow-band imaging at the same site. The villous structure is disappeared, and the marginal crypt epithelium appears curved shape. $\mathbf{c}$ Histopathological findings of the same site. Alcian blue-periodic acid Schiff staining; the mucosal epithelium replaced with the gastric crypt epithelium is stained reddish-purple.

\section{Secondary endpoints}

Examples of C-WLI, M-NBI, and corresponding histological findings of the normal duodenal mucosa and the duodenal mucosa with GM are shown in $\boldsymbol{\nabla}$ Fig. $\mathbf{1}$ and $\boldsymbol{\nabla}$ Fig. $\mathbf{2}$, respectively. When the duodenal endoscopic findings on C-WLI were examined, mucosal lesions were detected in $66 \%$ of patients $(33 / 50)$ in the CD group and $36 \%$ of those $(18 / 50)$ in the UC group; a significant difference was observed $(P<0.001)(\triangleright$ Table 4$)$. Based on the endoscopic findings on C-WLI, the most commonly observed lesion color was red, and the most commonly detected morphological types were the flat and depressed types $(\bullet \mathbf{T a}-$ ble 4). In addition, the presence of GM was histologically confirmed in $72.2 \%$ of the biopsy specimens collected from the flat and depressed lesions. 
Table 4 Conventional endoscopic findings in the duodenal mucosa.

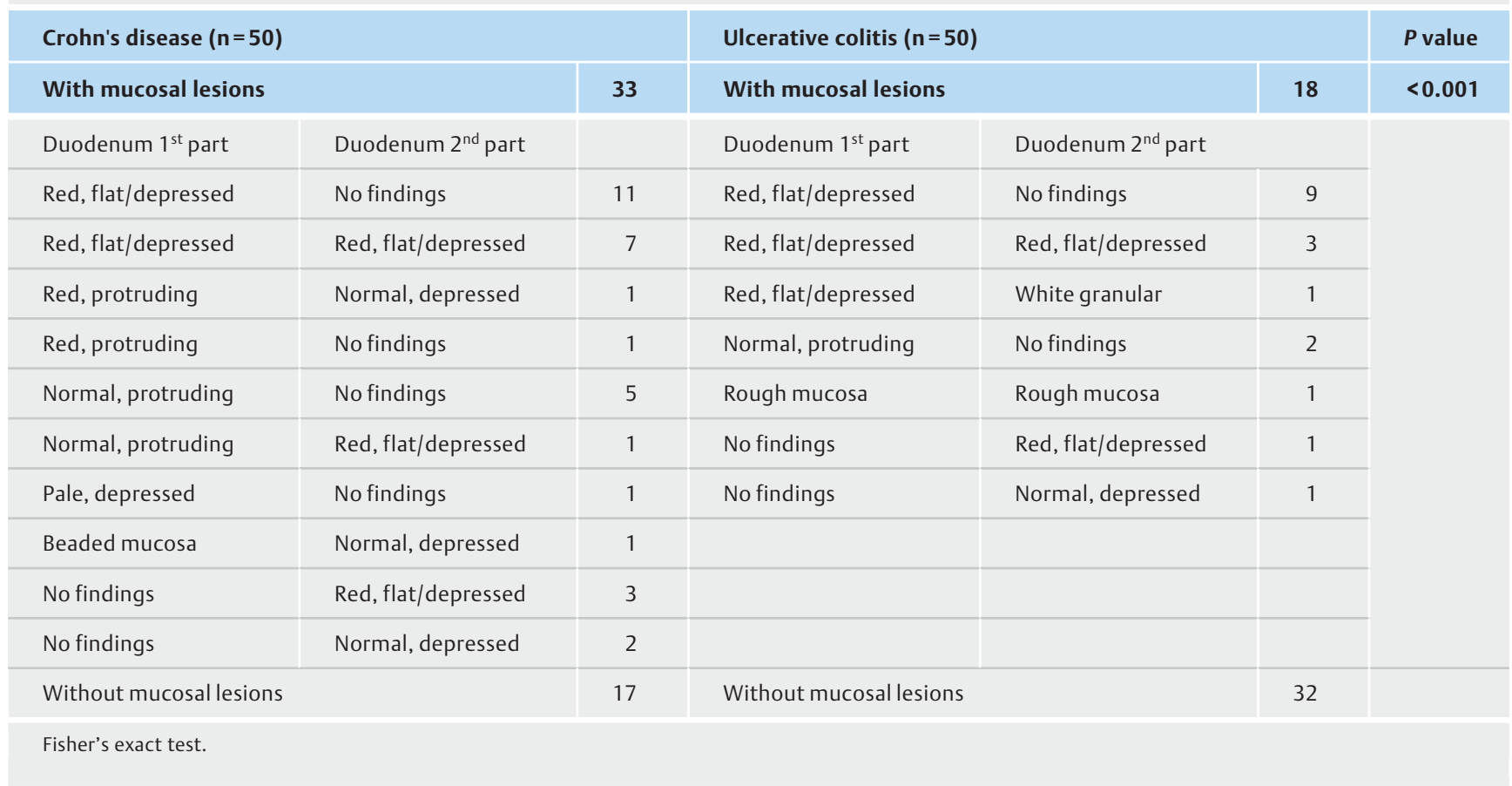

Table 5 Prevalence of gastric metaplasia in the duodenum according to presence or absence of a bamboo joint-like appearance $(n=50)$.

\begin{tabular}{|l|l|l|l|}
\hline & Gastric metaplasia & \multicolumn{1}{|c|}{ P value } \\
\hline Bamboo-joint-like appearance & Positive & Negative \\
\hline & Positive $(n=25)$ & $11(22 \%)$ & $14(28 \%)$ \\
\hline n.s., not significant; Fisher's exact test. & Negative $(n=25)$ & $13(26 \%)$ & $12(24 \%)$ \\
\hline
\end{tabular}

We found no significant difference in the frequency of GM between patients with and without a bamboo joint-like appearance or duodenal notch on C-WLI ( $\triangleright$ Table 5).

Duodenal mucosal lesions were endoscopically identified in 23 of 24 patients with histologically proven GM in the duodenum. The prevalence rates of GM were significantly different between the patients with and without mucosal lesions $(P<0.001)$ ( $\triangleright$ Table 6$)$. When the presence of mucosal lesions on C-WLI was used as an indicator of CD, C-WLI had a sensitivity of $95.8 \%$, a specificity of $61.5 \%$, and an accuracy of $78.0 \%$ for diagnosing GM ( $\triangleright$ Table 6 ).

A detailed review of M-NBI findings revealed that the characteristic features of GM were the disappearance of normal villous structure in the duodenal mucosa and the presence of curveshaped marginal crypt epithelium (MCE) at the site of the disappearance of the normal villous structure. These two features were termed magnifying endoscopic findings characteristic of gastric metaplasia (M-GM). In 22 of 24 patients with histologically proven GM in the duodenum, M-NBI showed findings characteristic of M-GM. The prevalence of GM was significantly higher in patients with M-GM findings than in those without them $(P<0.001)$ ( $\triangleright$ Table 7$)$. The following types of GM were noted: $26 \%$ (13/50), small- and large-intestine type, $10 \%(5 / 50)$ were small-intestine type; and $12 \%$ (6/50), large-intestine type ( $\triangleright$ Table 8). Meanwhile, the following types of M-GM were observed: $24 \%$ (12/50), small- and large-intestine type; $10 \%$ (5/50), small-intestine type; and $12 \%$ (6/50), large-intestine type ( $\triangleright$ Table 9 ).

When the presence of M-GM findings on M-NBI was used as an indicator of CD, M-NBI had a sensitivity of $91.6 \%$, a specificity of $96.2 \%$, and an accuracy of $94.0 \%$ for diagnosing GM ( $>$ Table 10). When M-NBI was performed in combination with C-WLI, significant increases were observed in the specificity and accuracy. M-NBI was found to have an additive effect. ( $\triangleright$ Table 11).

The diagnostic concordance rate between the two endoscopists for the presence of mucosal lesions on C-WLI was 83/ $100(83 \%)$, with a kappa coefficient of 0.68 (moderate). The diagnostic concordance rate between the two endoscopists for the presence of M-GM on M-NBI was 94/100 (94\%), with a kappa coefficient of 0.84 (excellent). The intra-observer concordance rate of $\mathrm{G}$. I . for the presence of mucosal lesions on CWLI for CD and UC was 93/100 (93\%), with a kappa coefficient of 0.86 (excellent). The intraobserver concordance rate of G.I. 
Table6 Prevalence of gastric metaplasia in the duodenum according to presence or absence of a duodenal notch $(n=50)$

\begin{tabular}{|c|c|c|c|c|}
\hline & & \multicolumn{2}{|c|}{ Gastric metaplasia } & \multirow[t]{2}{*}{$P$ value } \\
\hline & & Positive & Negative & \\
\hline \multirow[t]{2}{*}{ Duodenal notch } & Positive $(n=6)$ & $3(6 \%)$ & $3(6 \%)$ & n.s. \\
\hline & Negative $(n=44)$ & $21(42 \%)$ & $23(46 \%)$ & \\
\hline
\end{tabular}

- Table 7 Prevalence of gastric metaplasia in the duodenum of patients with Crohn's disease according to presence or absence of mucosal lesions detected by conventional white-light imaging.

\begin{tabular}{|c|c|c|c|c|}
\hline & & \multicolumn{2}{|c|}{ Gastric metaplasia } & \multirow[t]{2}{*}{$P$ value } \\
\hline & & Positive & Negative & \\
\hline \multirow[t]{2}{*}{ Mucosal lesion } & Positive $(n=33)$ & $23(46 \%)$ & $10(20 \%)$ & $<0.001$ \\
\hline & Negative $(n=17)$ & $1(2 \%)$ & $16(32 \%)$ & \\
\hline
\end{tabular}
Table 8 Prevalence of gastric metaplasia in the duodenum of patients with Crohn's disease according to presence or absence of magnifying endo-
scopic findings characteristic of gastric metaplasia on magnifying endoscopy with narrow-band imaging $(n=50)$.

\begin{tabular}{|c|c|c|c|c|}
\hline & & \multicolumn{2}{|c|}{ Gastric metaplasia } & \multirow[t]{2}{*}{$P$ value } \\
\hline & & Positive & Negative & \\
\hline \multirow[t]{2}{*}{ M-GM } & Positive $(n=23)$ & $22(44 \%)$ & $1(2 \%)$ & $<0.001$ \\
\hline & Negative $(n=27)$ & $2(4 \%)$ & $25(50 \%)$ & \\
\hline
\end{tabular}

Table 9 Prevalence of gastric metaplasia by type of Crohn's disease $(n=50)$

\begin{tabular}{|l|c|c|c|}
\hline & Gastric metaplasia & P value \\
\hline & Positive & Negative \\
\hline Small and large intestine & $13(26 \%)$ & $20(40 \%)$ & n.s. \\
\hline Small intestine & $5(10 \%)$ & $3(6 \%)$ \\
\hline Large intestine & $6(12 \%)$ & $3(6 \%)$ & \\
\hline n.s., not significant; Fisher's exact test. & & & \\
\hline
\end{tabular}

for the presence of M-GM on M-NBI for CD was 49/50 (98\%), with a kappa coefficient of 0.96 (excellent).

\section{Discussion}

In this study, the prevalence of GM of the duodenal mucosa was significantly higher in patients with CD who did not have HP infection than in control patients with UC. In this study, we histologically examined biopsy specimens collected from the gastric and duodenal mucosae of patients with CD who did not have HP infection and revealed that GM was often detected in the duodenum of patients with $C D$.
Metaplasia is a condition in which persistent mucous membrane disorders, such as chronic inflammation, induce the differentiation of already differentiated tissues into those with different properties. HP infection is generally reported to be a factor that causes GM of the duodenal mucosa [14]. To the best our knowledge, to date, no study has identified the presence of GM in the duodenal mucosa of patients with chronic inflammatory bowel diseases, including $C D$, based on histological findings. For the first time, this study revealed that GM occurs commonly in the duodenal mucosa of patients with CD who do not have HP infection, suggesting that the occurrence of GM in the duodenum may be a novel specific histopathological marker of CD. When our patients' endoscopic findings were exam- 
- Table 10 Prevalence of M-GM by type of Crohn's disease $(n=50)$.

\begin{tabular}{|l|l|l|l|}
\hline & M-GM & P value \\
\hline & Positive & Negative \\
\hline Small and large intestine & $12(24 \%)$ & $21(42 \%)$ & n.s. \\
\hline Small intestine & $5(10 \%)$ & $3(6 \%)$ \\
\hline Large intestine & $6(12 \%)$ & $3(6 \%)$ \\
\hline M-GM, magnifying endoscopic finding characteristic for gastric metaplasia; n.s., not significant; Fisher's exact test \\
\hline
\end{tabular}

- Table 11 Diagnostic performance of endoscopy in the diagnosis of gastric metaplasia in the duodenum of patients with Crohn's disease.

\begin{tabular}{|l|l|l|l|}
\hline & C-WLI (95\% Cl) & C-WLI+ M-NBI (95\% Cl) & P value \\
\hline Sensitivity & $95.8(90.1-99.9)$ & $91.6(83.5-99.1)$ & n.s. \\
\hline Specificity & $61.5(50.0-76.4)$ & $96.2(90.9-99.9)$ & 0.004 \\
\hline Accuracy & $78.0(66.5-89.5)$ & $94.0(93.6-94.4)$ & 0.039 \\
\hline C-WLI, conventional endoscopy with white-light imaging; M-NBI, magnifying endoscopy with narrow-band imaging; n.s., not significant; McNemar's test. \\
\hline
\end{tabular}

ined, the prevalence of mucosal lesions was significantly higher in the CD group than in the UC group $(P<0.001)$. As a bamboojoint-like appearance and duodenal notch have been reported to be characteristic C-WLI findings of CD [1-4], we also investigated their relationships with the presence of GM. However, there was no correlation between the presence of a bamboojoint-like appearance or duodenal notch and GM in the duodenum.

Next, the morphology of the lesions was examined on endoscopic images. In general, many endoscopic images of GM show protrusion [15].

In this study, GM was detected in $72.2 \%$ of the biopsy specimens collected from flat and depressed lesions. One of the reasons for this is that although GM owing to HP infection is an inflammatory condition that predominantly affects the mucosal epithelium in the superficial layer, CD is characterized by patchy transmural inflammation. When the inflammation affects the superficial layer, lesions may appear as focal flat lesions [5]. We also examined M-NBI findings and found that mucosa with histologically proven GM was characterized based on the findings of villous structure loss and the presence of arc-like structures in the MCE (M-GM). These microscopic focal M-GM findings may reflect noncontinuous patchy lesions of chronic inflammation in the mucosa that are caused by $C D$.

When M-NBI was performed together with C-WLI, significant increases were observed in specificity and accuracy. M-NBI was found to have an additive effect on C-WLI, and M-NBI showed a high diagnostic performance in terms of sensitivity, specificity, and accuracy. The use of M-NBI could have enabled the accurate and efficient diagnosis of GM in the duodenal mucosa. When we closely reexamined the histological findings of two patients with false-negative results on M-NBI, the area of GM in the biopsied tissues was very small. Therefore, we considered that M-GM findings could have been undetectable on magni- fied endoscopy. Furthermore, we closely reexamined the $\mathrm{M}$ $\mathrm{NBI}$ findings of patients with false-positive results and confirmed that the M-GM findings were certainly detectable. In other words, we assumed that the false-positive results were attributable to a sampling error because biopsy specimens were not accurately collected from the observation sites.

The interobserver concordance rate was calculated. The diagnostic concordance rate for mucosal lesions on C-WLI was $83.0 \%$ (kappa coefficient 0.68 [moderate]), whereas the diagnostic concordance rate for M-GM on M-NBI for CD was $94.0 \%$ (kappa coefficient 0.84 [excellent]). Accordingly, when mucosal lesions are detected on C-WLI, the presence of M-GM, confirmed by $\mathrm{M}-\mathrm{NBI}$, could be more reliable marker than that by $\mathrm{C}$ WLI for characterizing the detected lesion.

Based on the current study results, we propose a provisional strategy for the histological diagnosis of GM in the duodenum of Patients with CD who do not have HP infection ( $>$ Fig. 3). If the detection of mucosal lesions in the duodenum using C-WLI is followed by the detection of M-GM findings using $\mathrm{M}-\mathrm{NBI}$ in the same sites, a combination of C-WLI and M-NBI can be regarded as optical biopsy, in which GM is diagnosed based on endoscopic findings alone with a high diagnostic performance. When $\mathrm{M}-\mathrm{NBI}$ reveals a lack of M-GM findings, target biopsy of the mucosal lesions detected using C-WLI should be performed because the incidence of GM is higher in sites with mucosal lesions than in those without mucosal lesions. In the absence of mucosal lesions, random biopsy should be performed. The usefulness of this provisional strategy needs to be verified in a future prospective study. The histological findings of the presence of GM and the characteristic endoscopic findings of GM in the duodenum of HP-uninfected CD patient has not been described in previously published papers.

In the histopathological examination of biopsy specimens collected from the gastrointestinal mucosa (the stomach, duo- 


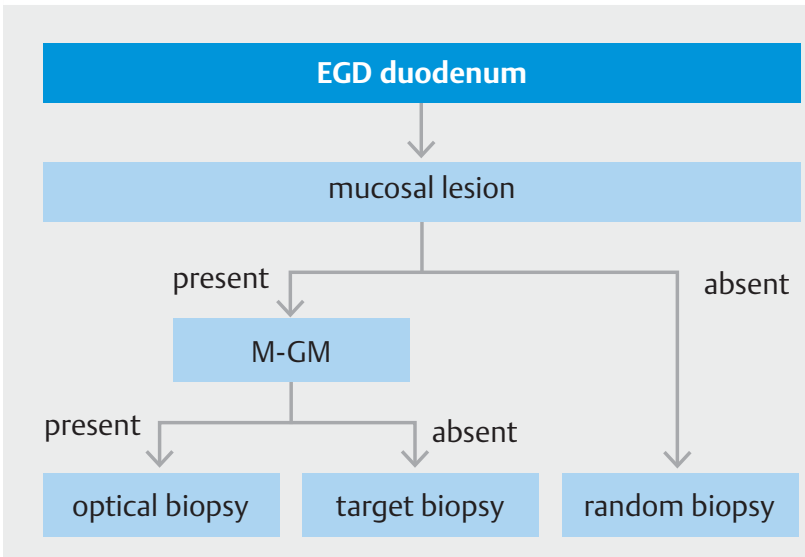

- Fig. 3 Provisional strategy for a more efficient histological diagnosis of gastric metaplasia in the duodenum. EGD, esophagogastoroduodenoscopy; M-GM, magnifying endoscopic finding characteristic of gastric metaplasia.

denum, small intestine, and large intestine) of patients with $C D$ during endoscopy, the presence of a noncaseating epithelioid granuloma is a characteristic finding. The detection rate of this finding by biopsy has been reported to range from approximately $26 \%$ to $67 \%[16,17]$. However, we recently observed a decline in the detection rate of noncaseating epithelioid granulomas. In fact, as demonstrated in this study, the prevalence rate of noncaseating epithelioid granulomas was only $2 \%$ and $2 \%$ in the stomach and duodenum, respectively.

In this study, we also performed histological and endoscopic examinations of patients' gastric mucosa. Intestinal metaplasia was detected in HP-uninfected gastric mucosae; however, its prevalence did not differ significantly between patients with $\mathrm{CD}$ and UC ( $\triangleright$ Table 3$)$. Although intestinal metaplasia is associated with HP infection $[18,19]$, to our knowledge, this study is the first to demonstrate that intestinal metaplasia occurs even in HP-uninfected gastric mucosae. Half of the gastric mucosal lesions exhibiting intestinal metaplasia appeared as red, patchy, shallow depressed lesions on C-WLI and were characterized by light blue crests on M-NBI. Furthermore, focally enhanced gastritis was histopathologically detected only in patients with $C D$. When the endoscopic findings of the sites showing histologically proven focally enhanced gastritis were reviewed, no characteristic endoscopic findings were identified.

At our hospital, upper gastrointestinal endoscopy, including that used for screening malignant tumors, is always performed with an endoscopic system using NBI and an optical magnifying endoscope [20]. Furthermore, after informed consent is obtained from patients with chronic inflammatory bowel disease, upper gastrointestinal endoscopy is performed according to the procedure used in this study. These clinical background characteristics allowed for the conduct of this retrospective study.

One of the limitations is that healthy controls were not included to our study. In addition, owing to the retrospective nature of this study, our results need to be verified in a future multicenter prospective study including healthy subjects.

\section{Conclusion}

In conclusion, histologically proven GM of the duodenal mucosa was observed more commonly in patients with CD who did not have HP infection than in patients with UC. The identified endoscopic findings may be useful as novel indicators for the histological diagnosis of GM in the duodenum.

\section{Competing interests}

The authors declare that they have no conflict of interest.

\section{References}

[1] Yao T, Iwashita A. Upper gastrointestinal involvements of Crohn's disease. Stomach Intest (Tokyo) 1983; 18: 1323-1334 (in Japanese, with English abstract)

[2] Yokota K, Saito Y, Einami K et al. bamboo joint-like appearance of the gastric body and cardia: possible association with Crohn's disease. Gastrointest Endosc 1997; 46: 268-272

[3] Sakatani A, Fujiya M, Kashima S et al. Upper-gastrointestinal Lesions in Patients with Inflammatory Bowel Diseases. Stomach Intest (Tokyo) 2013; 48: 636-644 (in Japanese, with English abstract)

[4] Yoshiki N, Kentaro M, Mikihiro F et al. The endoscopic findings of the upper gastrointestinal tract in patients with Crohn's disease. Clin J Gastoroenterol 2017; 10: 289-296

[5] Iwashita A, Yamada Y, Yao T et al. Pathology for Crohn's disease. Stomach Intest (Tokyo) 1997; 32: 365-376 (in Japanese, with English abstract)

[6] Oberhuber G. Focally enhanced gastritis: A frequent type gastritis in patients with Crohn's disease. Gastroenterology 1997; 112: 698-706

[7] Akemoto Y, Sakuraba H, Tanaka M et al. Gastric focal neutrophil infiltration and wide duodenal gastric foveolar metaplasia are histologic discriminative markers for Crohn's disease and Behcet's disease. Digestion 2019; 100: 210-219

[8] Kimura K, Takemoto T. An endoscopic recognition of the atrophic border and its significance in chronic gastritis. Endoscopy 1969; 1 : 87-97

[9] Saskaki N, Momma K, Egawa N et al. The influence of Helicobacter pylori infection on the progression of gastric mucosal atrophy and occurrence of gastric cancer. Eur J Gastroenterol Hepatol (Suppl I) 1995: S59-62

[10] Oken MM, Creec RH et al. Toxicity and response criteria Of The Eastern Cooperative Oncology Group. Am J Clin Oncol 1982; 5: 649-655

[11] Gormally SM, Kierce BM, Daly LE et al. Gastric metaplasia and duodenal ulcer disease in children infected by Helicobacter pylori. Gut 1996; 38: 513-517

[12] Voutilanen M, Juhola M, Farkkila M et al. Gastric metaplasia and chronic inflammation at the duodenal bulb mucosa. DigestLiver Dis 2003; 35: 94-98

[13] Landis JR, Koch GG. The measurement of observer agreement for categorical data. Biometrics 1977; 33: 159-174

[14] Shirai T. The pathogenetic role of gastric metaplasia and Helicobacter Pylori duodenal ulcer. Gastroenterol Endosc 1991; 33: 2177-2182 (in Japanese, with English abstract)

[15] Nakai H, koizumi I, Saionnji K. Relation to the background gastric mucosa and Helicobacter pylori infections. Gastroenterol Endosc 1995; 37: 529-539 (in Japanese, with English abstract) 
[16] De Matos V, Russo PA, Cohen AB et al. Frequency and clinical correlations of granulomas in children with Crohn's disease. J Pediatr Gastroenterol Nutr 2008; 46: 392-398

[17] Rubio CA, Orrego A, Nesi G et al. Frequency of epithelioid granulomas in colonoscopic biopsy specimens from paediatric and adult patients with Crohn's colitis. J Clin Pathol 2007; 60: 1268-1272

[18] Mutoh H, Hakamata Y, Sato K et al. Conversion of gastric mucosa to intestinal metaplasia in Cdx2-expressing transgenic mice. Biochem Biophys Res Commun 2002; 294: 470-479
[19] Rita AB, Alex B. Intestinal metaplasia: A premalignant lesion involved in gastric carcinogenesis. J Gastroenterol Hepatol 2009; 24: 193-201

[20] Yao K, Doyama H, Gotoda T et al. Diagnostic performance and limitations of magnifying narrow-band imaging in screening endoscopy of early gastric cancer: a prospective multicenter feasibility study. Gastric Cancer 2014; 17: 669-679 